\title{
Calming the cytokine storm in COVID-19
}

\author{
Cytokine blockade can improve the survival rate of patients with COVID-19 who are at risk of respiratory failure, but \\ timing and patient selection are key.
}

Randy Q. Cron, Roberto Caricchio and W. Winn Chatham

$\mathrm{T}$ he coronavirus SARS-CoV-2 has infected more than 200 million people and killed over 4 million, with a mortality rate of approximately $2 \%$ worldwide. Many who die from COVID-19 suffer hyper-inflammation with features of cytokine storm syndrome (CSS) and associated acute respiratory distress syndrome ${ }^{1,2}$. Although the antiviral remdesivir was shown to reduce the length of hospital stay for those with COVID $-19^{3}$, only anti-inflammatory approaches have improved survival in these patients. The greatest survival benefit has been demonstrated with broadly immunosuppressive glucocorticoids when given to those with an oxygen requirement ${ }^{4}$. However, in the absence of an oxygen requirement ${ }^{4}$ or substantial systemic inflammation (as measured by C-reactive protein (CRP) $)^{5}$, patients treated with glucocorticoids may fare worse than those who receive standard care. Thus, the selection of patients and timing of the administration of glucocorticoids is critical for survival benefit. Ideally, earlier use of more-targeted anti-cytokine therapy to prevent CSS, without increasing viral replication, is needed.

In this issue of Nature Medicine, Kyriazopoulou et al. report their evaluation of blockade of the cytokine IL-1 via the recombinant human IL-1 receptor antagonist anakinra in patients with COVID-196. Almost 600 patients with COVID-19 pneumonia who were at risk for respiratory failure, as defined by an elevated serum level of soluble urokinase-type plasminogen activator receptor (suPAR), were randomly assigned to receive either anakinra or placebo (along with standard of care) early during hospitalization, before they needed mechanical ventilation. Perhaps the elevated suPAR level identified not only those with excess inflammation but also those with organ damage such as renal injury or cardiovascular occlusion due to COVID-19-associated thrombosis. Over $80 \%$ of patients in the placebo and anakinra arms also received standard-of-care glucocorticoids, yet anakinra still notably improved survival and shortened hospital stay.

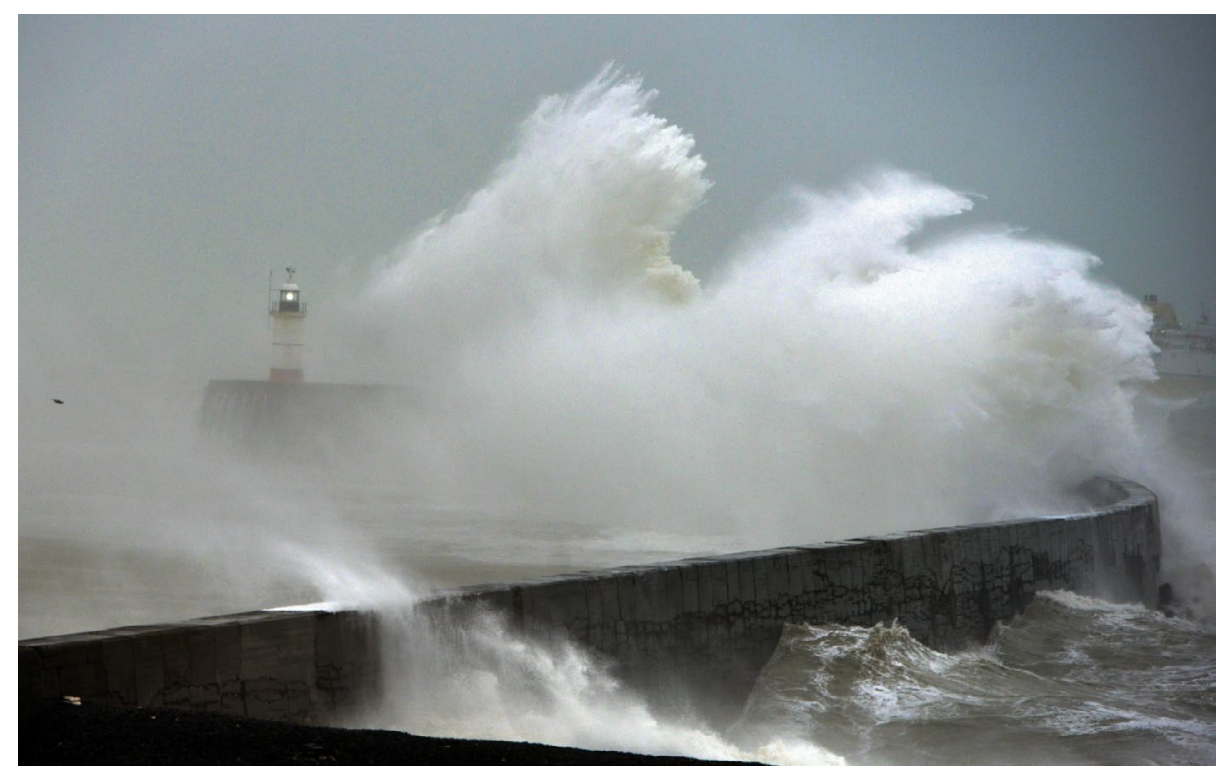

Credit: Peter Cripps / Alamy Stock Photo

This is in contrast to a recent randomized, double-blind, placebo-controlled trial of canakinumab, a monoclonal antibody to IL- $1 \beta$, in treating 454 hospitalized, hypoxemic patients with COVID-19 pneumonia before mechanical ventilation ${ }^{7}$. An elevated level of CRP or ferritin, each a marker of CSS, was required for study entry. Although there was a trend toward improved survival in the canakinumab arm, and a substantially greater number of patients in the placebo arm received IL- 6 blockers or anakinra after randomization, the study did not demonstrate a significantly greater likelihood of survival for those who received canakiumab. There are some key differences between these trials, one being that anakinra blocks signaling of both IL- $1 \alpha$ and IL-1 $\beta$, whereas canakinumab binds only IL-1 $\beta$. SARS-CoV-2-infected endothelium may be a particularly important source of IL- $1 \alpha$ that is not targeted by canakinumab. In addition, the selection of patients by suPAR levels rather than by CRP or ferritin levels may better define those patients with COVID-19 pneumonia most likely to benefit from IL-1 blockade.

Another cytokine-targeted approach to treating severe COVID-19 includes IL-6 blockade with monoclonal antibodies to either IL- 6 or the IL- 6 receptor. There have been many reported clinical trials exploring inhibition of IL-6 for the treatment of COVID-19; no survival benefit was demonstrated in randomized, placebo-controlled, blinded trials, whereas, on the other hand, large, non-blinded, randomized studies have supported a survival benefit with IL-6 blockade ${ }^{8}$. Meta-analyses also suggest some modest benefit overall with this approach, and again selection of patients and timing of administration are likely to be key factors ${ }^{8}$. Most recently, a randomized, placebo-controlled trial of Janus-kinase inhibition has been reported to improve COVID-19 survival, in the presence of background glucocorticoid treatment (received by $89 \%$ of patients) ${ }^{9}$. Janus-kinase inhibition targets multiple inflammatory cytokines that use common signaling 
pathways employed by shared cytokine receptors and thus is intermediate between broadly immunosuppressive glucocorticoids and individually targeted cytokine therapeutics.

Knowing which cytokine or cytokines to target in severe COVID-19 pneumonia remains a conundrum. One thing that is clearly emerging from this pandemic is that the CSS associated with COVID-19 is relatively unique, with only modestly elevated levels of IL-6, CRP and ferritin, for example. Studies exploring serum biomarkers clearly distinguish COVID-19induced CSS from more classic CSS such as macrophage-activation syndrome or hemophagocytic lymphohistiocytosis ${ }^{10}$. Conceivably, some of the relatively lower cytokine levels in patients with COVID-19 CSS may be related to the profound lymphopenia seen in these patients. By comparison, deadly strains of influenza virus, perhaps including the virus that caused the 1918 influenza pandemic, can lead to a more classic CSS ${ }^{11}$. Nevertheless, inhibition of the hyper-inflammatory immune response in severe COVID-19 pneumonia undoubtedly improves the survival of patients, particularly through glucocorticoids given to those with oxygen requirements. Timing of the administration of immunosuppressive or immunomodulatory agents is key-such that suppressing the host response too early during rapid viral replication is probably deleterious, whereas requirement for respiratory support may be the appropriate time to intervene. More-targeted approaches such as anakinra, however, may allow earlier introduction of anti-cytokine treatment.

As this pandemic stretches on, researchers and clinicians will continue to hone their knowledge of how best to treat patients hospitalized with severe COVID-19 pneumonia in order to improve survival rates. Current approaches include antivirals, proning and CSS therapies, among others. Beyond glucocorticoids, it remains to be seen precisely which targeted approaches are likely to be effective in treating the unique CSS induced by COVID-19. The finding of elevated levels of endogenous antagonist to the IL-1 receptor in patients with severe COVID-19 suggests that there is an IL-1 gene signature ${ }^{10}$. Perhaps IL-1 blockade with anakinra in select patients with COVID-19 pneumonia will gain traction as further data emerge. In the meantime, it is important to continue efforts to prevent the spread and development of severe SARS-CoV-2 infection with expanded availability and administration of safe and effective vaccines ${ }^{12}$.
Randy Q. Cron ${ }^{1 凶}$, Roberto Caricchio ${ }^{2}$ and W. Winn Chatham

${ }^{1}$ Division of Clinical Immunology and Rheumatology,

University of Alabama School of Medicine,

Birmingham, AL, USA. ${ }^{2}$ Section of Rheumatology,

Lewis Katz School of Medicine, Temple University,

Philadelphia, PA, USA.

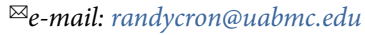

Published online: 3 September 2021

https://doi.org/10.1038/s41591-021-01500-9

References

1. Cron, R. Q. \& Chatham, W. W. J. Rheumatol. 47, 639-642 (2020).

2. Fajgenbaum, D. C. \& June, C. H. N. Engl. J. Med. 383,

2255-2273 (2020).

3. Beigel, J. H. et al. N. Engl. J. Med. 383, 1813-1826 (2020).

4. Horby, P. et al. N. Engl. J. Med. 384, 693-704 (2021).

5. Keller, M. J. et al. J. Hosp. Med. 15, 489-493 (2020).

6. Kyriazopoulou, E. et al. Nat. Med. https://doi.org/10.1038/s41591 021-01499-z (2021).

7. Caricchio, R. et al. J. Am. Med. Assoc. 326, 230-239 (2021).

8. Shankar-Hari, M. et al. J. Am. Med. Assoc. 326, 499-518 (2021).

9. Guimaraes, P. O. et al. N. Engl. J. Med. 385, 406-415 (2021)

10. Kessel, C. et al. Arthritis Rheumatol. https://doi.org/10.1002/ art.41763 (2021).

11. Schulert, G. S. et al. J. Infect. Dis. 213, 1180-1188 (2016)

12. Fauci, A. S. Science 372, 109 (2021).

Competing interests

R.Q.C. has served as a consultant to Sobi, Novartis, Pfizer and Sironax. R.Q.C. and W.W.C. have received grant support from Sobi for investigator-initiated clinical trials. R.C. has served as a consultant to GlaxoSmithKline, Johnson \& Johnson, Aurinia and Bristol Myers Squibb.

\title{
Anemia in women - an intractable problem that requires innovative solutions
}

\author{
A new spatial analysis calls for a tiered and geographically targeted approach to tackling the vast, ongoing burden \\ of anemia in women within low- and middle-income countries.
}

\section{Parul Christian}

\footnotetext{
A nemia in women of reproductive age (15-49 years of age) in low- and middle-income countries (LMICs) continues to be an intractable problem of 'hidden hunger', exemplifying gender health inequities and a shameful loss of human capital. The low hemoglobin concentration in blood that defines anemia occurs long after tissue iron stores have been depleted to levels associated with suboptimal function. A major cause of anemia, this iron deficiency leads to diminished oxygen-carrying capacity in red blood
}

cells, which in turn diminishes energy efficiency, work capacity and productivity ${ }^{1}$. In addition, severe anemia in pregnancy is consistently linked to maternal mortality ${ }^{2}$. Anemia in women of reproductive age is concentrated in LMICs and requires urgent and immediate attention. In this issue of Nature Medicine, Kinyoki and colleagues set out to model the prevalence and burden of mild, moderate and severe anemia in LMICs over the past two decades ${ }^{3}$.

The study examines data from several databases across 82 LMICs, comprising
218 geo-referenced household surveys conducted between 2000 and 2018 and including over three million women. On the basis of their analysis, the authors estimate half a billion women were living with anemia in LMICs in 2018. In the period from 2000 to 2018, they find limited decreases in anemia prevalence $(35.6 \%$ to $31.6 \%)$ and an increase in years lived with disability, due to population growth. They reveal widespread sub-national disparities and predict that a vast majority of countries will fail to achieve the World Health 